\title{
Phytoöstrogene - wie ist der Erkenntnisstand?
}

Phytoöstrogene sind pflanzliche Phenole, deren chemische Struktur eine gewisse Verwandtschaft zu 17-beta-Estradiol aufweist. Man kennt im Wesentlichen vier verschiedene Verbindungsgruppen, die diese Verwandtschaft zeigen: die Isoflavone, die Lignane, die Coumestane und die Stilbene. Lignane sind im Pflanzenreich weit verbreitet, besonders konzentriert in Leinsamen, Broccoli und manchen Getreiden. Isoflavone sind als Inhaltsstoffe von Soja und Rotklee besonders bekannt und liegen dort überwiegend als Glykoside vor. Coumestane und Stilbene haben bisher - mit Ausnahme des im Rotwein enthaltenen Resveratrols - eine geringere Aufmerksamkeit erlangt. Insgesamt sind heute mehrere hundert Pflanzenin-

Isoflavone können haltsstoffe mit östrogener Wirdas Brustkrebs- und kung bekannt. Die überwieOsteoporoserisiko gende Zahl der klinischen Stuvermindern dien mit Phytoöstrogenen befasst sich mit Kombinationen von Isoflavonen. Ausgangspunkt sind dabei epidemiologische Befunde aus Asien, die zeigen, dass in Populationen mit hohem Verzehr an Sojaprodukten die Inzidenzen an Brust-, Darm- und Prostatakrebs drastisch niedriger liegen als in Bevölkerungsgruppen mit westlicher Ernährung. Ebenso sind in diesen Populationen die Häufigkeit der Osteoporose und das Auftreten menopausaler Beschwerden wesentlich geringer. In der Behandlung menopausaler Beschwerden und zur Prophylaxe von Osteoporose sowie von Darmkrebs steht die Hormonersatztherapie aufgrund großer klinischer Studien ganz im Vordergrund. Allerdings ist damit ein leicht erhöhtes Risiko von Brustkrebs und thromboembolischen Episoden verbunden, sodass sich die Frage nach Alternativen bei der Behandlung menopausaler Beschwerden und Risiken stellt. Bei sojareicher Ernährung liegen die Plasmaspiegel an Isoflavonen ca. zwanzigmal höher als bei westlicher Ernährung, d.h. mehr als hundertmal höher als die Spiegel an 17-beta-Estradiol. Daidzein und Genistein, die wichtigsten bioaktiven Isoflavone, zeigen eine fünfzigmal geringere Bindungsaffinität an den Östrogenrezeptor, wobei sie eine deutlich höhere Affinität zum beta- als zum alfa-Östrogenrezeptor aufweisen, d.h. weder das Endometrium noch das Brustdrüsengewebe werden durch Isoflavone stimuliert.
Bei der Bewertung der klinischen Wirksamkeit der Phytoöstrogene zeigt eine erst kürzlich zusammen mit dem Deutschen Krebsforschungszentrum durchgeführte kontrollierte Studie, dass bei Frauen um die Menopause die regelmäßige Zufuhr von Isoflavonen und Lignanen in moderater Dosis das Brustkrebsrisiko vermindert. Ebenso zeigt die überwiegende Anzahl der Studien zur Protektion des Skeletts gegen Osteoporose eine positive Wirkung der Isoflavone, sodass die Empfehlung einer verstärkten Isoflavonzufuhr zur Vorbeugung gegen Osteoporose bei Risikopatientinnen angezeigt ist. Dabei sollte die Zufuhr in einer Tagesdosis von 50-100 mg bereits vor der Menopause beginnen. Soja ist von der amerikanischen FDA als wirksam in der Vorbeugung von Herzinfarkt anerkannt worden, was mit der Verbesserung der NO-Produktion des Gefäßendothels unter dem Einfluss von Isoflavonen zusammenhängt, sodass die periphere Durchblutung verbessert wird. Diese vaskulären Wirkungen dürften auch für die Besserung menopausaler Beschwerden verantwortlich sein, die in einer Reihe klinischer Studien nachgewiesen sind. Wichtig ist auch dabei natürlich der rechtzeitige prämenopausale Beginn der Einnahme. Um die positiven Wirkungen der Phytoöstrogene sicherzustellen, ist es besonders wichtig, dass der Isoflavongehalt der entsprechenden Produkte nachgewiesen ist, d.h. dass in dieser Richtung eine ausreichende Qualitätssicherung und Deklaration der Produkte stattfindet. Dies ist leider nicht immer der Fall. Ebenso beachtenswert ist der Einfluss der Darmflora auf die Bioverfügbarkeit der Nahrungsisoflavone, da sie aus den Glykosiden freigesetzt werden müssen, um in eine resorbierbare Form zu gelangen. So ist beispielsweise nach der Einnahme von Antibiotika die Resorption der Isoflavone drastisch verringert.

Bei Beachtung dieser wichtigen Zusammenhänge sind anhand der vorliegenden epidemiologischen, experimentellen und klinischen Befunde die Isoflavone als die wichtigste Gruppe der Phytoöstrogene besonders wertvolle Mikronährstoffe mit großem präventiven Potenzial. Weitere Forschung ist erforderlich, um den Erkenntnisstand über die vielen anderen Phytoöstrogene zu verbessern.

Karlheinz Schmidt 